Canad. Math. Bull. Vol. 44 (4), 2001 pp. 509-512

\title{
Index of Volume 44
}

A 257 Abánades, Miguel A. Algebraic Homology For Real Hyperelliptic and Real Projective Ruled Surfaces

3 Alexandru, Victor, Nicolae Popescu, Alexandru Zaharescu The Generating Degree of $\mathbb{C}_{p}$

12 Anisca, Razvan, Monica Ilie A Technique of Studying Sums of Central Cantor Sets

B 385 Ballantine, Cristina M. A Hypergraph with Commuting Partial Laplacians

19 Brindza, B., Á. Pintér, W. M. Schmidt Multiplicities of Binary Recurrences

C 398 Cardon, David A., M. Ram Murty Exponents of Class Groups of Quadratic Function Fields over Finite Fields

266 Cencelj, M., A. N. Dranishnikov Extension of Maps to Nilpotent Spaces

270 Cheung, Wai-Shun, Chi-Kwong Li Linear Operators Preserving Generalized Numerical Ranges and Radii on Certain Triangular Algebras of Matrices

129 Currás-Bosch, Carlos Linéarisation symplectique en dimension 2

D 266 Dranishnikov, A. N. See M. Cencelj

E 22 Evans, Ronald Gauss Sums of Orders Six and Twelve

F 408 Falbel, E. Finite Groups Generated by Involutions on Lagrangian Planes of $\mathbf{C}^{2}$

G 420 Gauthier, P. M., M. R. Pouryayevali Approximation by Meromorphic Functions with Mittag-Leffler Type Constraints 
27 Goodaire, Edgar G., César Polcino Milies Normal Subloops in the Integral Loop Ring of an RA Loop

140 Gotay, Mark J., Janusz Grabowski On Quantizing Nilpotent and Solvable Basic Algebras

140 Grabowski, Janusz See Mark J. Gotay

H 429 Henniger, J. P. Ergodic Rotations of Nilmanifolds Conjugate to Their Inverses 440 Hironaka, Eriko The Lehmer Polynomial and Pretzel Links

I 12 Ilie, Monica See Razvan Anisca 452 Ishihara, Hironobu Some Adjunction Properties of Ample Vector Bundles

J 150 Jakóbczak, Piotr Exceptional Sets of Slices for Functions From the Bergman Space in the Ball

K $459 \mathrm{Kahl}$, Thomas LS-catégorie algébrique et attachement de cellules

36 Kapovich, Michael, John J. Millson Quantization of Bending Deformations of Polygons In $\mathbb{E}^{3}$, Hypergeometric Integrals and the Gassner Representation

61 Kats, B. A. The Inequalities for Polynomials and Integration over Fractal Arcs

L 160 Langlands, Robert P. The Trace Formula and Its Applications: An Introduction to the Work of James Arthur

282 Lee, Min Ho, Hyo Chul Myung Hecke Operators on Jacobi-like Forms

70 Lempert, László, Róbert Szőke The Tangent Bundle of an Almost Complex Manifold

210 Leung, Man Chun Growth Estimates on Positive Solutions of the Equation $\Delta u+K u^{\frac{n+2}{n-2}}=0$ in $\mathbb{R}^{n}$

80 Levin, Michael Constructing Compacta of Different Extensional Dimensions

$270 \mathrm{Li}$, Chi-Kwong See Wai-Shun Cheung

87 Lieman, Daniel, Igor Shparlinski On a New Exponential Sum

M 469 Marcoux, Laurent W. Sums and Products of Weighted Shifts 
223 Marshall, $M$. Extending the Archimedean Positivstellensatz to the Non-Compact Case

292 McKay, Angela An Analogue of Napoleon's Theorem in the Hyperbolic Plane

482 Mezo, Paul Matching of Weighted Orbital Integrals for Metaplectic Correspondences

27 Milies, César Polcino See Edgar G. Goodaire

36 Millson, John J. See Michael Kapovich

298 Muić, Goran A Proof of Casselman-Shahidi's Conjecture for Quasi-split Classical Groups

398 Murty, M. Ram See David A. Cardon

282 Myung, Hyo Chul See Min Ho Lee

N 93 Neumann, B. H. Some Semigroup Laws in Groups

O $97 \mathrm{Ou}$, Zhiming M., Kenneth S. Williams On the Density of Cyclic Quartic Fields

P 105 Pilipović, Stevan Convolution Equation in $\mathcal{S}^{\prime *}$ - Propagation of Singularities

19 Pintér, Á. See B. Brindza

3 Popescu, Nicolae See Victor Alexandru

420 Pouryayevali, M. R. See P. M. Gauthier

R 313 Reverter, Amadeu, Núria Vila Images of mod $p$ Galois Representations Associated to Elliptic Curves

231 Rosenblatt, Joseph M., George A. Willis Weak Convergence Is Not Strong Convergence For Amenable Groups

115 Roy, Damien Approximation algébrique simultanée de nombres de Liouville

S 19 Schmidt, W. M. See B. Brindza

242 Schueller, Laura Mann The Zeta Function of a Pair of Quadratic Forms

323 Schuman, Bertrand Une classe d'hamiltoniens polynomiaux isochrones

87 Shparlinski, Igor See Daniel Lieman

335 Stacey, P. J. Inductive Limit Toral Automorphisms of Irrational Rotation Algebras

70 Szőke, Róbert See László Lempert 
V 313 Vila, Núria See Amadeu Reverter

337 Vinet, Luc, Alexei Zhedanov Spectral Transformations of the Laurent Biorthogonal Polynomials, II. Pastro Polynomials

W 346 Wang, Wei Positive Solution of a Subelliptic Nonlinear Equation on the Heisenberg Group

491 Wang, Weiqiang Resolution of Singularities of Null Cones

355 Weaver, Nik Hilbert Bimodules with Involution

370 Weston, Anthony On Locating Isometric $\ell_{1}^{(n)}$

97 Williams, Kenneth S. See Zhiming M. Ou

231 Willis, George A. See Joseph M. Rosenblatt

121 Wojciechowski, Michat A Necessary Condition for Multipliers of Weak Type $(1,1)$

Z 3 Zaharescu, Alexandru See Victor Alexandru 126 Zeron, E. Santillan Each Copy of the Real Line in $\mathbb{C}^{2}$ is Removable 376 Zhang, $X i$ A Note on $p$-Harmonic 1-Forms on Complete Manifolds 504 Zhang, Yong Weak Amenability of a Class of Banach Algebras 337 Zhedanov, Alexei See Luc Vinet 